Bull. Mater. Sci., Vol. 22, No. 5, August 1999, pp. 877-884. O Indian Academy of Sciences.

\title{
Computational condensed matter physics
}

\author{
B K GODWAL \\ Condensed Matter Physics Division, Bhabha Atomic Research Centre, Mumbai 400 085, India
}

\begin{abstract}
In the high pressure laboratory at BARC, we are pursuing a program to study the behaviour of materials under static and dynamic pressures. Theoretical component has been an integral part for guiding and interpreting new experiments. The initial phase of such efforts was devoted to the development of equation of state models at arbitrary temperatures and matter densities. With the advent of diamond anvil cell device and the simultaneous provision for laser heating of the compressed samples for static high pressure studies, and with the improvements of the diagnostic techniques in dynamic shock methods, the focus of our studies switched over to the predictions and interpretations of phase transitions. Often these efforts have led to intense experimental studies and sometimes helped in resolving the controversies in data. We adopted the first principles electronic structure calculations for high pressure studies. Our work on the electronic topological transition in zinc led to many experimental and theoretical investigations. The results of electronic structure changes in similar metal cadmium shall be compared with existing understanding in Zn under pressure. Our studies on $\mathrm{Nb}$ and other compounds like intermetallics and borocarbides have revealed interesting electronic structure changes under pressure. However, the electronic structure based investigations of structural stabilities at high pressures involve tedious trial and error effort, which is avoided in the ab initio molecular dynamics simulations. The current status of our efforts in the use of this technique is illustrated with the example of quasicrystal based clusters.
\end{abstract}

Keywords. Pressure; electronic topological transition; equation of state.

\section{Introduction}

Pressure as a thermodynamic variable in condensed matter studies has become very important in recent past. In fact the research at high pressures has truely developed into an interdisciplinary area which has important implications for and in the areas of physics, chemistry, materials science, biology, engineering and technology. The main effect of pressure is to reduce the volume of a substance. Thus in some sense, it mimics the phenomena taking place during the cohesion of solids. Therefore significant changes are expected, and indeed found, in the electronic states, chemical bonding, and atomic packing of condensed matter (Jeanloz 1987; Mao and Hemley 1994).

In the high pressure physics laboratory at BARC we have been pursuing a program to investigate materials for their physical properties at high pressures with special emphasis on equation of state (EOS) and phase stability using both static and dynamic techniques (Godwal 1995; Chidambaram 1996; Sikka et al 1997). A brief mention of our past work on EOS will be made with the current trends in theoretical methods to study EOS and phase transitions. The introduction of linear methods in electron energy band theory and availability of supercomputers, or parallel processors as an alternative, have given boost to the computational physics. The current efforts are on the generalized gradient approximation (GGA) based $a b$ initio electronic structure calculations and molecular dynamics (MD) simulations. Our work on electronic structure will be illustrated in the present article, especially where our theoretical investigations have played important roles in the interpretation of experimental data, like resolving the discrepancies, understanding of the mechanism for the observed phase transitions [Th] and predictions and finding the cause of anomalies in physical properties [ $\left.\mathrm{Zn}, \mathrm{Cd}, \mathrm{Nb}, \mathrm{AuIn}_{2}, \mathrm{YNi}_{2} \mathrm{~B}_{2} \mathrm{C}\right]$, etc. Finally, our simulations by $a b$ initio molecular dynamics will be presented. These first principles MD simulations compared to band structure computations have a promise to avoid the tedious search of various structures for structural stability by trial and error in phase transition studies under pressure or temperature. We have employed both the orbital based (Car-Parrinello method) and density based $a b$ initio MD simulations (Pearson et al 1993; Shah et al 1994; Smargiassi and Madden 1994) in our calculations. The current status of our efforts in the use of this technique is illustrated with the example of $\mathrm{Al}-\mathrm{Zn}-$ $\mathrm{Mg}$ quasicrystal based systems like clusters and crystal approximants to investigate the crucial role played by glue atoms in propagating the icosahedral symmetry.

\section{Equation of state (EOS)}

One of the most important outputs of high pressure theory and experiments is the pressure-volume-temperature 
relationship of materials termed the EOS, the utility of which for meaningful interpretation of physical and chemical phenomena at arbitrary temperatures and matter densities is well known (Chidambaram 1996; Sikka et al 1997). At extremely high temperatures or high pressures (for pressures in few hundreds of Megabars range), the various variants of statistical Thomas-Fermi-Dirac theories are adequate to generate the EOS (McCarthy 1965). For the pressures up to $1000 \mathrm{GPa}$ we adopted the usual division of the internal energy and pressure into temperature independent and dependent contributions

$$
\begin{aligned}
& E=E_{\mathrm{c}}(V)+E_{\mathrm{ion}}(V, T)+E_{\mathrm{ex}}(V, T), \\
& P=P_{\mathrm{c}}(V)+P_{\mathrm{ion}}(V, \Psi)+P_{\mathrm{ex}}(V, T) .
\end{aligned}
$$

Here the symbols c, ion and ex stand for cold $(0 \mathrm{~K})$, lattice thermal vibrations and electronic excitations, respectively. The zero degree isotherm is evaluated using density functional based electron band structure methods with the atomic numbers and crystal structure as input (Godwal et al 1983; Sikka et al 1997). The lattice vibration contribution in quasi-harmonic approximation is computed by Born-von Karman lattice dynamic theory and the electronic excitation term derived from low temperature expansions of Fermi-Dirac distribution function (Godwal et al 1983). The total energy and pressure evaluated using these equations are used in Rankine-Hugoniot relations to calculate the shock Hugoniot which represent the locii of all the points which can be reached by shocking a material from fixed initial conditions. (Zel'dowich and Raizer 1967; Chidambaram 1996). Early on, we successfully calculated the shock Hugoniot equation of state of $\mathrm{Al}$ and were able to discriminate the two sets of shock data (Godwal et al 1979). These calculations were extended to many elements of interest for which the extensive shock data exist (Godwal et al 1983).

For the pressures in 1000 to $10000 \mathrm{GPa}$ range, pressure and thermal ionization effects strongly controlled by atomic shell structure effects start dominating many physical properties. The orthogonality condition which keeps the core and conduction electrons apart also gets violated due to core ionization. The basic problem in this region is to obtain core ionization and core-conduction electron coupling and the resulting modified potential. For this difficult region which is known as intermediate region' in the literature the experimental data were sparse earlier and, in the absence of any existing theory, interpolation schemes were used between the experimental (pressures less than $1000 \mathrm{GPa}$ ) and high density Thomas-Fermi-Dirac region (McCarthy 1965). We approached this region from the solutions of the details of the ionization equilibrium by including the high density effects like pressure ionization, partition function cut off and lowering of the ionization potentials in Saha ionization theory (Rouse 1971; Godwal 1983, 1995a, b). With new electronic charge and potential parameters the condensed matter theories were then employed for the calculation of shock Hugoniot EOS (Godwal et al 1981). In the absence of pressure and thermal ionization effects, this theory coincided with the model in the experimental region while in the high density region its predictions coincided with those of Thomas-Fermi-Dirac theories (McCarthy 1965). Many of the predictions made by this theory were later verified by the experiments (Volkov et al 1980; Godwal et al 1983, 1990).

\section{Phase transitions}

Pressure induced phase transitions have been the most important area of research with compression of about half easily achieved in most of the materials using the DAC. Hence the high pressure phase transitions have been extensively studied both theoretically and experimentally (Jayaraman 1983; Chidambaram 1996; Sikka et al 1997). The $a b$ initio total energy calculations and first principles MD simulations have been going through rapid expansions during the last decade. The aim of these calculations have been to predict accurately structures and related ground state properties of solids without using any information from experiments. Hence they are ideally suited for the predictions of phase transitions, especially under the thermodynamic conditions not yet accessible to the laboratory experiments. We present below briefly some of our experimental and theoretical work on elemental solids ( $\mathrm{Th}, \mathrm{Zn}, \mathrm{Cd}, \mathrm{Nb}$ ), and compounds $\left(\mathrm{AuIn}_{2}\right.$, and $\mathrm{YNi}_{2} \mathrm{~B}_{2} \mathrm{C}$ ) which emphasize the usefulness of the electronic structure calculations on identifying the underlying physical mechanism for the observed experimental phenomena, especially in the case of the electronic topological transitions (ETT) (Lifshitz 1960).

\subsection{Elemental solids}

3.1a Thorium: Thorium crystallizes in the face centred cubic ( $\mathrm{fcc}$ ) structure at ambient conditions. Vohra and Akella (1991) found that it undergoes fcc to body centred tetragonal (bct) structural phase transition around $100 \mathrm{GPa}$ pressure. With the help of energy band structure calculations by the linear muffin-tin-orbital (LMTO) method (Andersen 1975; Skriver 1984; Christensen 1985), Rao et al (1992) studied the $5 f$ band characteristic through the fcc-to-bct transition and found that the bottom of the band falls below the Fermi level $\left(E_{\mathrm{F}}\right)$ at the volume fraction of 0.6 (around $80 \mathrm{GPa}$ pressure). It was thus established that the $f$-band occupation is central to this structural transition. It was also shown, by carrying out additional total energy calculations by restricting the LMTO expansion of the electron wave function up to angular momentum $1=2$ that $\mathrm{Th}$ is not spd-metal as 
thought earlier, but it has contribution to bonding from the $5 f$ band even at ambient pressure through hybridization. The exclusion of $1=3$ term led to stabilization of the body-centred cubic (bcc) phase at ambient pressure, which continued to remain stable to the volume fraction of $V / N_{0}=0.4$. These findings were later re-confirmed by Johansson and coworkers (1995) using full potential (FPLMTO) calculations. Our theoretical isotherms with and without inclusion of $5 f$ electrons are compared in figure 1 with the experimental data of Vohra and Holzapfel (1993). The observed fcc structure and the EOS are consistent with the view that $\mathrm{Th}$ should be considered as spdf-metal belonging to heavier actinide group $(\mathrm{Pa}-\mathrm{Pu})$, and not as spd-transition metal belonging to the same group as $\mathrm{Ti}, \mathrm{Zr}$ and $\mathrm{Hf}$ ( $\mathrm{Rao}$ et al 1992; Sikka et al 1997).

3.1b Zinc and cadmium: Zinc and cadmium are unique among hcp metals, having unusually large $c / a$ axial ratio at ambient condition $(c / a=1.856$ and 1.886 , respectively). The large deviation from the ideal $c / a$ value of 1.633 causes anisotropies in many of their physical properties, some of which are attributed to the changes in the Fermi surface (FS). Both these metals exhibit giant Kohn anomaly (GKA) at ambient pressure (Kohn 1959; Kagan et al 1983). The application of pressure alters the FS topology, transport and other physical properties, and have led to intense theoretical and experimental studies of these materials under compression (Meenakshi et al 1992; Potzel et al 1995; Takemura 1995; Morgan et al 1996).

The anomaly in the variation of the axial ratio and electrical resistance of $\mathrm{Zn}$ with pressure was first reported

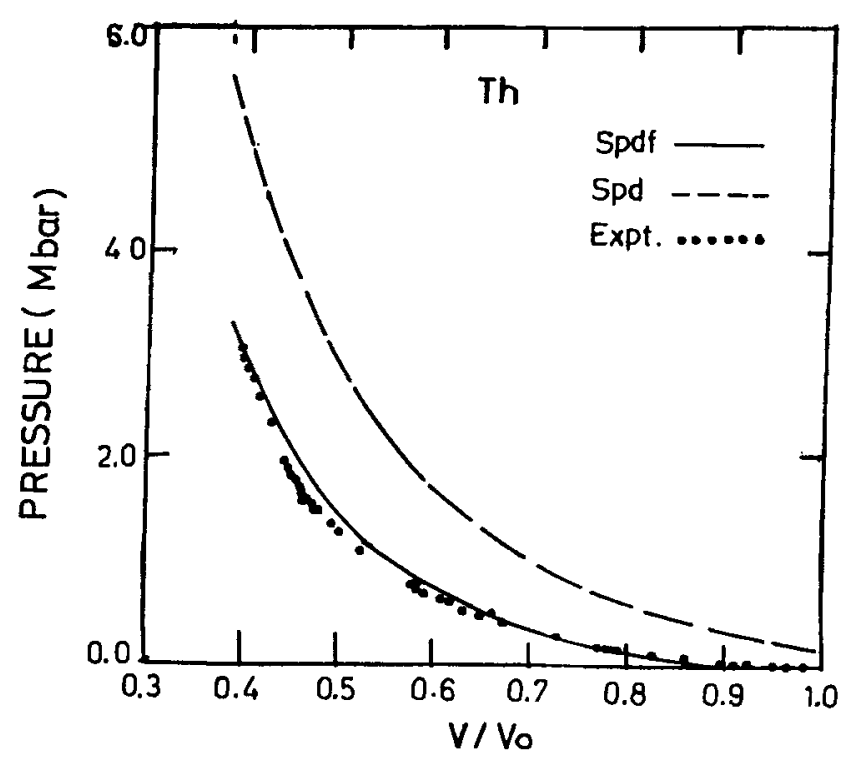

Figure 1. $0 \mathrm{~K}$ isotherm of thorium. The curve referred as spd is for the case when f-orbital is suppressed in the calculation. Experimental data (Vohra and Holzapfel 1993) are shown for comparison. around $7 \mathrm{GPa}$ (Lynch and Drickamer 1965), but a later experiment could not find the anomaly up to $32 \mathrm{GPa}$ (Schulte et al 1991). Due to this controversy, we calculated the change in the axial ratio for $\mathrm{Zn}$ under pressure from the first principles total energy calculations based on the LMTO method (Meenakshi et al 1992). These calculations showed that volume dependence of the axial ratio changes slope around the relative volume of 0.92 , and the details of the electronic band structure revealed that the anomaly is related to the appearance of maximum in the density of states (DOS) at $E_{\mathrm{F}}$. Takemura (1995) later observed that the volume dependence of the $c / a$ ratio changes slope at $V / N_{0}=0.893$. Also Potzel et al (1995) have recently detected an anomaly in the Mössbauer spectrum of $\mathrm{Zn}$ at $6.6 \mathrm{GPa}$ and $4.2 \mathrm{~K}$. Their scalar-relativistic linear augmented plane wave calculations verified that the anomaly is related to the topological change of the FS around the $L$ symmetry point of the Brillouin zone (BZ) and at this pressure it leads to the destruction of the GKA (Potzel et al 1995; Steiner et al 1996). Moreover, the anomaly observed in the recent neutron inelastic scattering experiments has also been interpreted in the similar way (Morgan et al 1996). However, the occurrence of the anomaly in $\mathrm{Zn}$ in the high pressure angle-dispersive $\mathrm{X}$-ray diffraction (ADXRD) experiments (Takemura 1995) at the $\sqrt{3}$ value of $(c / a)$, for which significant degeneracies in the reciprocal lattice vectors occur for the hcp structure, cast doubts on ETT as the sole cause of the anomaly. In order to remove this ambiguity we undertook studies on similar metal $\mathrm{Cd}$ as it also exhibits GKA at normal pressure. The details are reported elsewhere (Godwal et al 1997c), and we briefly summarize the key results below.

We employed the same theoretical formalism as used for $\mathrm{Zn}$ (Meenakshi et al 1992) and predicted two anomalies in $\mathrm{Cd}$ (see figure 2). The first anomaly is around $V / V_{0}=0.95$ although it is not observed in the experimental data (Lynch and Drickamer 1965; Schulte et al 1991; Takemura 1997). However, around this pressure, our measurement of thermoelectric power (TEP) showed a broad maximum (Vijayakumar et al 1997), and based on our LMTO calculations, this corresponds to appearance of additional K-point needles in the FS (third zone electron sheet). The second anomaly occurs at $V N_{0}=0.85$ and is also seen in the recent experimental data of Takemura (1997), and also in the earlier data of Lynch and Drickamer (1965), but not in the work of Schulte et al (1991). However, at this pressure, there is no ETT to destroy the GKA although theoretical calculations support the presence of ETT in Zn (Meenakshi et al 1992; Potzel et al 1995; Steiner et al 1996). This reveals that electronic structure of $\mathrm{Cd}$ under pressure is different from that of $\mathrm{Zn}$, which is in contrast to the currently speculated view that the $\mathrm{FS}$ topologies of $\mathrm{Zn}$ and $\mathrm{Cd}$ under pressure are identical. Our calculations show a continuous and slow transfer of electrons from $d$-like to $s$-like states 
under compression, and the turn-over in the $4 d$ partial pressure contribution to bonding from negative (filled band) to positive around this anomaly due to increase in the $d-d$ overlap. Moreover, when we analyzed using universal EOS, the experimental data on $\mathrm{Zn}$ (Takemura 1995) and Cd (Takemura 1997), it showed deviations from linearity in $\mathrm{Zn}$ at the observed anomaly, and in $\mathrm{Cd}$ around the first anomaly. These findings are consistent with the view that the universal EOS should show deviation from linearity whenever an electronic transition occurs (Sikka 1988). Note that our prediction of two anomalies in $\mathrm{Cd}$ is consistent with the later FPLMTOGGA calculations of Novikov et al (1997) who find anomalies in the $c / a$ ratio at $V / V_{0}=0.95$ and 0.85 .

Uncertainties still exist in $\mathrm{Zn}$ regarding ETT as the cause of anomaly in $c / a$ variation with pressure. Based on the recent LDA electronic structure calculations, Fast $e t$ al (1997) claim that the electronic states around the K-point of the $\mathrm{BZ}$ are responsible for the observed anomaly. Also, Novikov et al (1997) based on the FPLMTO-GGA calculations find the anomalies in the pressure dependence of $c / a$ near experimental values for both $\mathrm{Zn}$ and $\mathrm{Cd}$, and also obtain similar anomalies in electric field gradient. However, their studies do not support the destruction of GKA due to ETT. On the experimental side, the recent high pressure room temperature neutron inelastic experiments of Klotz et al (1998) up to $9.4 \mathrm{GPa}$, do not support the ETT and destruction of GKA. However, the need of the hour is to carry out the high pressure experiments at very low temperatures to reduce the smearing of the Fermi distribution function which dampens the anomalies due to ETT. It will be ideal to

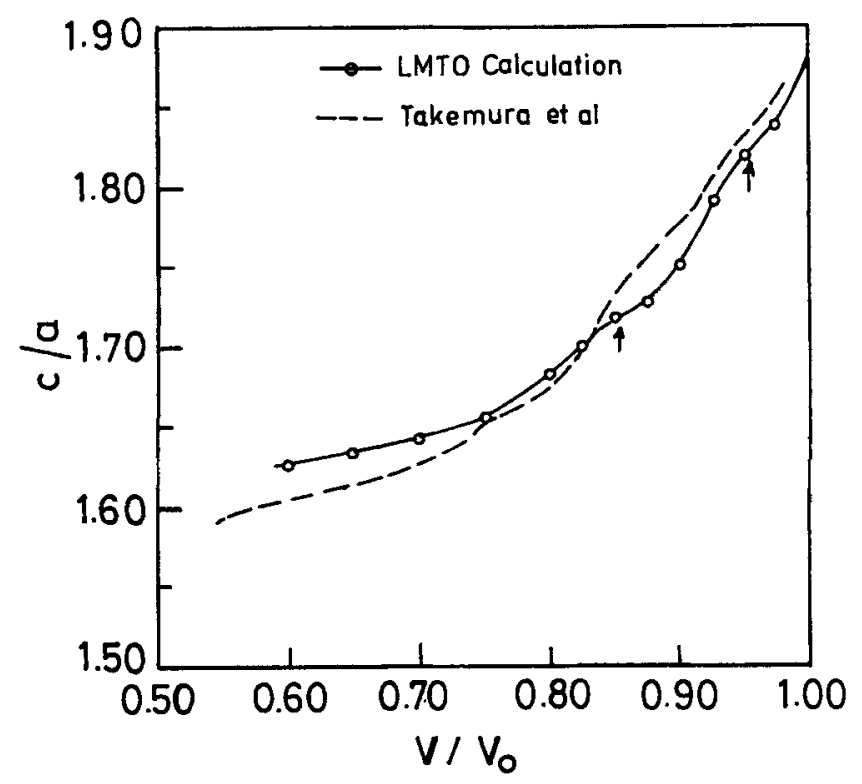

Figure 2. Comparison of the calculated (c/a) variation with relative volume compression $\left(V / V_{0}\right)$ and the experimental data (Takemura 1997) for cadmium. carry out the high pressure Fermi surface measurements at low temperatures to identify directly the possibility of ETT under pressure in $\mathbf{Z n}$.

3.1c Niobium: The group VB transition metals such as vanadium, niobium and tantalum are particularly interesting because of their superconducting properties, and for the anomalies in the phonon dispersion curves. Fermi surface topologies of the VB group transition metals were studied by Mattheiss (1970) using non self-consistent augmented plane-wave method. The effect of pressure on the electronic structure of niobium was first studied by Anderson et al (1981) by the self-consistent augmented plane-wave method. They found that Fermi surfaces of $\mathrm{Nb}$ at $6 \mathrm{GPa}$, and at $26 \mathrm{GPa}$ are similar. Recent superconducting transition temperature $\left(T_{\mathrm{c}}\right)$ measurements under compression up to megabar pressures (Struzhkin et al 1997) using highly sensitive magnetic susceptibility technique show two anomalies in $\mathrm{Nb}$, one near $5 \mathrm{GPa}$ and the other in the 60-70 $\mathrm{GPa}$ range. However, the high pressure static and dynamic experiments of Akahama et al (1991) show that the ambient pressure body centred cubic (bcc) structure of $\mathrm{Nb}$ is stable up to $170 \mathrm{GPa}$. Hence any change in $T_{c}$ may mainly be due to changes in the electronic structure. Motivated by these observations, we have carried out the electronic structure calculations of $\mathrm{Nb}$ in the bcc phase as a function of pressure.

We employed the first principles full potential linear muffin tin orbital (FPLMTO) method as per Methfessel's (1988) version. Hedin-Lundquist $(1971,1972)$ local density approximation (LDA) was employed.

The calculated electronic band structure at normal pressure is similar to those available in the literature (Mattheiss 1970; Anderson et al 1981). Accordingly, the energy bands of $\mathrm{Nb}$ are similar to those of other group $\mathrm{VB}$ transition metals, $\mathrm{V}$ and $\mathrm{Ta}$, the main differences being the width and position of the $d$ bands, and the $s-d$ band separation. We have calculated the total energies by changing atomic volume from $133.5 \mathrm{a} \cdot \mathrm{u}^{3}$ to $85 \mathrm{a} \cdot \mathrm{u}^{3}$. The equation of state (EOS) of $\mathrm{Nb}$ obtained by using these total energies (not shown here and the details will be published elsewhere, (Palanivel et al 1999)) agrees well with the experimental data of Akahama et al (1991) as well as with shock data (Kinslow 1970). The details of electron energy bands have also been studied at different compressed volumes up to the volume corresponding to about $60 \mathrm{GPa}$ pressure.

To explain the observed anomaly in $T_{\mathrm{c}}$ of $\mathrm{Nb}$ at $5 \mathrm{GPa}$, Struzhkin et al (1997) very recently have suggested that it is due to change in the Fermi surface topology due to shift of a band near Fermi energy $\left(E_{\mathrm{F}}\right)$. According to them, the two third zone Fermi surface sheets may touch to form a neck along $\Gamma N(\Sigma)$ direction at ambient pressure, which means that the minimum of the third band along $\Sigma$ is at higher energy than $E_{\mathrm{F}}$. They then attribute the $T_{\mathrm{c}}$ anomaly to the disappearance of this neck as the $d$-band lowers 
with respect to $E_{\mathrm{F}}$ under compression, and touches it at $5 \mathrm{GPa}$. The present FPLMTO calculations show that the minimum of the relevant band (see figure 3a) is below, $E_{\mathrm{F}}$, and thus the necks do not exist in $\mathrm{Nb}$ at normal pressure. We also found that the shift of this band with respect to $E_{\mathrm{F}}$ along $\Sigma$ is relatively small in the pressure range of 0 to $5 \mathrm{GPa}$. Note that there is no other experimental evidence of these necks in $\mathrm{Nb}$.

Thus we conclude that in contrast to the suggestion of Struzhkin et al (1997), the experimentally observed anomaly in $T_{\mathrm{c}}$ at $5 \mathrm{GPa}$ is not due to ETT. This anomaly may be due to uniaxial stresses in the sample, which is also the alternative suggestion given by Struzhkin et al (1997). Note that the $d$ energy level $\Gamma_{25}^{\prime}$ crosses $E_{\mathrm{F}}$ at a volume compression $V / V_{0}$ of about 0.8 (see figure $3 \mathrm{~b}$ ), which corresponds to about $60 \mathrm{GPa}$ pressure. This ETT is in good agreement with the second anomaly in $T_{c}$ observed experimentally (Struzhkin et al 1997) in the 60$70 \mathrm{GPa}$ pressure range, and supports the interpretation of Struzhkin et al (1997).

\subsection{Intermetallics}

3.2a $A u l n_{2}:$ We are carrying out a program to investigate intermetallics for their high pressure behaviour by using both theory and experiments. In particular, our interest in $\operatorname{AuIn}_{2}$ is due to the observed anomaly of a change in the slope of the melting curve near $3 \mathrm{GPa}$ pressure (Storm et al 1996), which generally indicates a phase transition. As many of the halides and oxides pertaining to the geophysical interest occur in the same structure, phase transformation studies of $\mathrm{AuIn}_{2}$ under compression are useful to give indications of high pressure phases of these oxides and halides. Motivated by these factors, we carried out the ADXRD (with imaging plate), electrical resistivity, and TEP measurements on
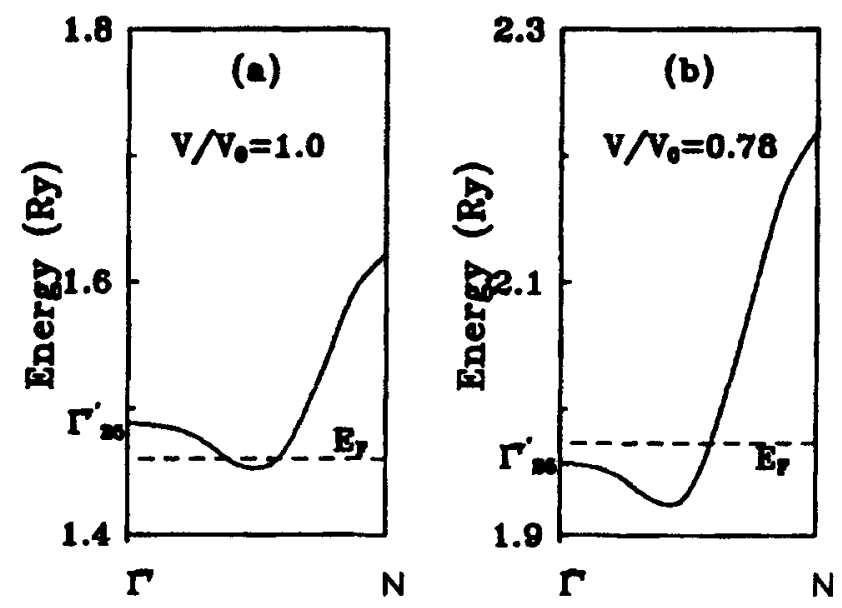

Figure 3. Electron energy band along $\Gamma N(\Sigma)$ in $N b$ at (a) ambient pressure, and (b) at volume compression $V / V_{0}$ of 0.78 . The broken lines denote the respective Fermi levels $\left(E_{\mathrm{F}}\right)$.
AuIn $_{2}$ (Godwal et al 1997a). The electrical resistivity is structureless, though decreases rapidly and the sign of the slope changes near $3 \mathrm{GPa}$, whereas the TEP increases rapidly near $2 \mathrm{GPa}$ pressure. But, the ADXRD measurements rule out the structural changes up to $8 \mathrm{GPa}$. Thus the isostructural electronic structure changes appear to be responsible for the observed anomalies. For further insight, we carried out the LMTO calculations in the $\mathrm{CaF}_{2}$ structure under compression. The details of the calculations were presented elsewhere (Godwal et al 1997a). A significant result obtained is the crossing of an extremum (maximum) of an electron energy band through Fermi level $\left(E_{\mathrm{F}}\right)$ as pressure varies (see figure 4a). Also, our universal EOS (Rose et al 1984) estimates show deviations from linearity in the 2-4 GPa pressure range (figure 4b) indicating electronic transition (Sikka 1988). Thus our electronic structure calculations reveal that the anomalies in the melting curve and the transport properties are due to ETT.
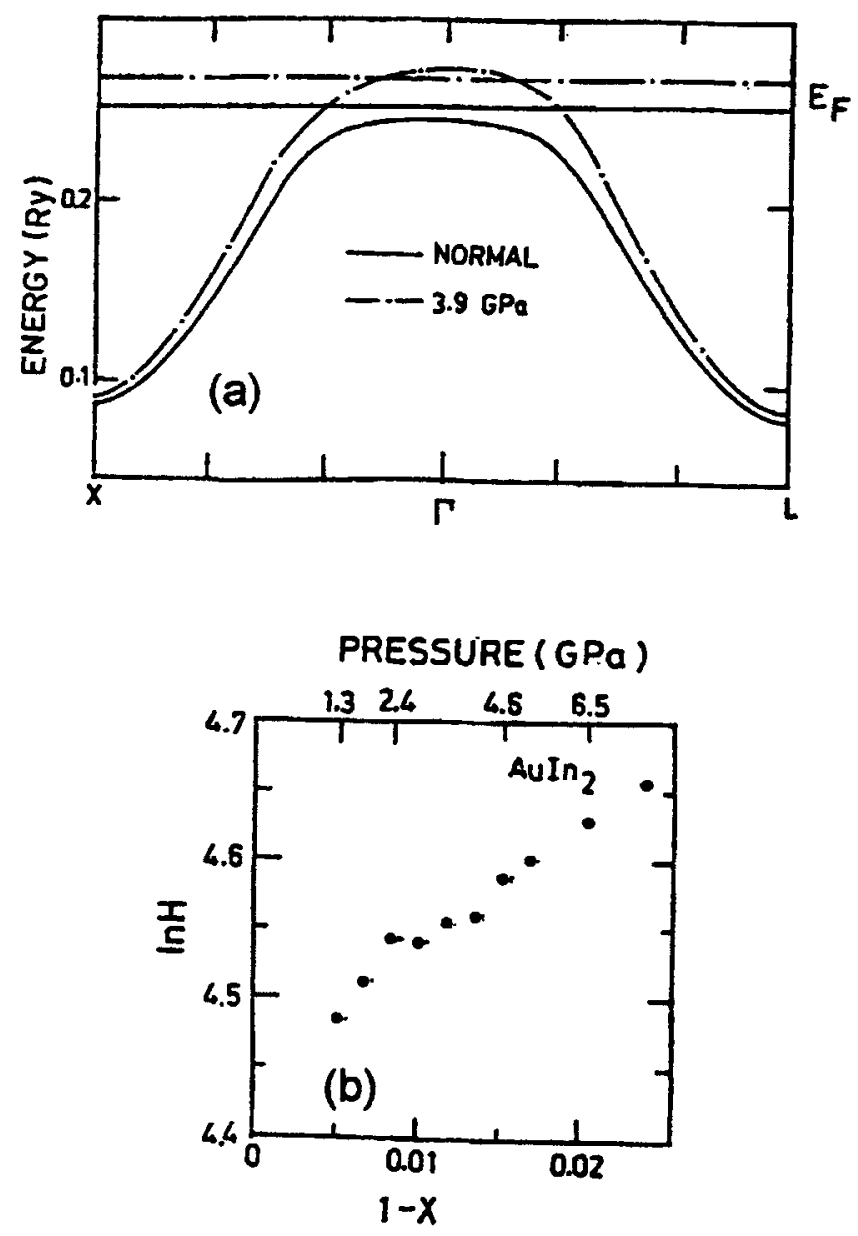

Figure 4. (a) Shift of the energy band maximum at $\Gamma_{2}{ }^{\prime}$ across the Fermi level $\left(E_{\mathrm{F}}\right)$ from the occupied to the unoccupied region under pressure; (b) the universal equation of state for $\mathrm{AuIn}_{2}$ by electronic structure calculations. Departure from linearity in the curve occurs in the pressure range of 2 to $4 \mathrm{GPa}$. The pressure values at a few compressions are shown. 
3.2b $\mathrm{YNi}_{2} \mathrm{~B}_{2} \mathrm{C}$ : The new family of borocarbide superconductors have been extensively investigated recently (Mazumdar et al 1993; Cava et al 1994; Nagarajan et al 1994; Meenakshi et al 1996). Some of their distinct properties are: short B-C separation (1.46 $\AA$ ), near ideal tetrahedral coordination of $\mathrm{Ni}$ and $\mathrm{B}$ atoms, and short $\mathrm{Ni}-\mathrm{Ni}$ separation $(2.45 \AA)$, i.e. shorter than in Ni-metal. Also, the variation of their superconducting transition temperature $\left(T_{\mathrm{c}}\right)$ with pressure is interesting as most of the lanthanide borocarbides show linear dependence, with the slope changing sign between $\mathrm{Lu}$ and $\mathrm{Tm}$ in the series. The high pressure electronic structure studies are desirable as they might identify the features in the electronic spectrum which lead to these systematic changes in $T_{\mathrm{c}}$ with pressure. Also, as these changes affect the transport properties like electrical resistance and thermoelectric power (TEP), we also carried out these measurements under pressure. Moreover, we measured the ADXRD at high pressures to look for any structural change, and to obtain the EOS. The theoretical calculations were carried out by the tight binding LMTO (TB-LMTO) (Andersen and Jepsen 1984) and the FPLMTO methods (Wills and Cooper 1987).

We found rapid decrease in resistivity with pressure up to about $2 \mathrm{GPa}$, and a smooth weak pressure dependence thereafter up to $8 \mathrm{GPa}$, suggesting absence of structural transition. But the TEP measurements showed a peak in the 2-4 GPa pressure range, which indicates significant changes in the electronic DOS at $E_{\mathrm{F}}$. Absence of structural transition in this pressure region was further confirmed by our ADXRD measurements. We observed no structural phase change up to $16.4 \mathrm{GPa}$, and $\mathrm{YNi}_{2} \mathrm{~B}_{2} \mathrm{C}$ was found to retain its ambient phase; the carbon filled variant of $\mathrm{ThCr}_{2} \mathrm{Si}_{2}$ type tetragonal structure (space group $14 / \mathrm{mmm}$ ). The EOS obtained from the lattice parameter measurements with the ADXRD set up is shown in figure 5 . The measured $(P-V)$ data compare reasonably well with our TB-LMTO calculations. Both the experimental $P-V$ data and the theoretical estimates yielded the bulk modulus of $200 \mathrm{GPa}$. Also shown in figure 5, are the EOS curve from the FPLMTO (Meenakshi et al 1998) calculations which agree better with the experimental data. Moreover, the FPLMTO calculations on other borocarbide $\mathrm{LuNi}_{2} \mathrm{~B}_{2} \mathrm{C}$ also give almost coinciding curve with that of $\mathrm{YNi}_{2} \mathrm{~B}_{2} \mathrm{C}$. Our calculations also showed that the boron component of DOS is reduced at $E_{\mathrm{F}}$ on compression, apart from the usual band broadening effect, as the peak in the vicinity of $E_{\mathrm{F}}$ moves away from it (see Meenakshi et al 1998, for details). This feature would predict a fall of $T_{\mathrm{c}}$ with pressure in $\mathrm{YNi}_{2} \mathrm{~B}_{2} \mathrm{C}$ (Mattheiss $e t$ al 1994). However in the pressure range up to $2 \mathrm{GPa}$, the change in the boron component of DOS at $E_{\mathrm{F}}$, as obtained by our calculations, is so small that almost no change in $T_{\mathrm{c}}$ is expected. This is consistent with the available experimental data (Schmidt and Braun 1994; Alleno et al 1995).

\section{Ab initio molecular dynamics}

The first principles electronic structure based total energy calculations can determine the most stable structure at high pressures from the chosen candidate structures, but might fail to locate one of the lowest free energy, as the search is only by trial and error. The MD simulations can locate the structure giving global minimum energy by employing standard techniques like simulated annealing (Kirkpatrick et al 1983). However, the bonding characteristics generally could change drastically under pressure, and hence the $a b$ initio MD simulations would be preferred. But the coupled density functional MD calculations based on Kohn-Sham equations (Kohn and Sham 1965) demand unmanageable computer power for complex systems, as one has to carry out the calculations for unoccupied electron states though not used anywhere in the self-consistent process. This drawback reduces the efficiency of the computations by at least an order of magnitude. Alternatively, the direct minimization of total energy keeps track of occupied electron orbitals only. Starting with any arbitrary set of trial electron orbitals, one can iteratively upgrade them to get the minimum of total energy, and the set of orbitals at the minimum energy represents the Kohn-Sham solution. Further, to study the dynamics of ions, their positions can be included as additional variables and the structural relaxation can be treated as a global minimization problem. Car and Parrinello (1985) treated coefficients of electron wavefunctions and ionic positions as though they formed a dynamical system and the corresponding phase space is explored by MD simulations. The constant pressure (variable cell) simulations by this method can identify the high pressure phase transitions in solids (Focher et al 1994). We are adapting these methods for high pressure studies. The current status of our efforts in the use of this

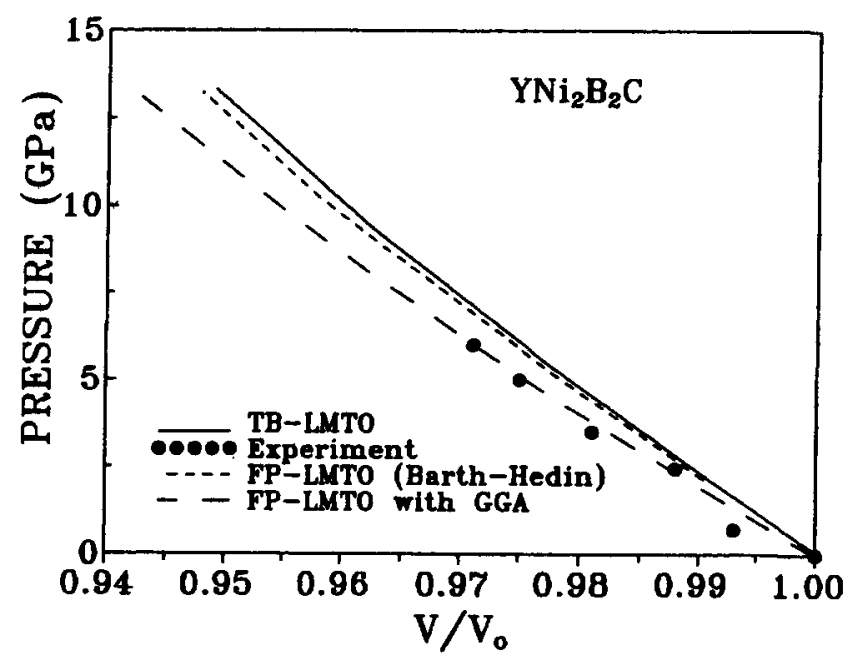

Figure 5. Equation of state for $\mathrm{YNi}_{2} \mathrm{~B}_{2} \mathrm{C}$. The curve for $\mathrm{LuNi}_{2} \mathrm{~B}_{2} \mathrm{C}$ by the FPLMTO calculations coincides with the dashed curve and hence is not shown separately. 
technique will be illustrated below by a few examples at ambient pressure by constant volume MD simulations. We have employed both the orbital based (Car and Parrinello 1985) and the density based (Pearson et al 1993; Shah et al 1994; Smargiassi and Madden 1994) methods in our $a b$ initio MD simulations, using the standard atomic pseudopotentials of Bachelet-Hamann-Schluter (Bachelet et al 1982).

\subsection{Orbital based ab initio $M D$}

The first method (Car-Parrinello method) is based on the plane wave expansion of valence electron orbitals. We have implemented this orbital based $a b$ initio MD scheme for constant volume simulations on BARC parallel processor ANUPAM. The program employs frequent transformations between reciprocal and direct spaces. Hence the direct and inverse fast Fourier transforms (FFTs) are parallelized (Jagadeesh et al 1996). By employing assembler coded FFTs, we get a speed-up of about 4 in the 8-node machine. We have applied parallelized C-P method to study the molten phase of sulphur. The calculated pair distribution function (Godwal et al 1997b) compares fairly well with the results of neutron diffraction experiments (Descotes and Bichara 1995). The constant pressure (variable cell) simulations are being implemented to compute thermodynamic properties and phase stability of materials.

\subsection{Density based ab initio $M D$}

The second method adopted in our calculations employs the plane wave expansion of the square root of total valence electron density (Pearson et al 1993; Shah et al 1994; Smargiassi and Madden 1994).

The density based MD simulations were carried out on clusters related to $\mathrm{Al}-\mathrm{Zn}-\mathrm{Mg}$ quasicrystal. $\mathrm{Al}$ was chosen at the centre of the cluster as our earlier study (Rao et al 1994) on the crystal approximants with $\mathrm{Zn}_{48} \mathrm{Mg}_{32}$ around different ( $\mathrm{Al}, \mathrm{Zn}, \mathrm{Mg}$ and empty) central atoms in a bcc crystal structure had shown that $\mathrm{Al}$ atom at the central site would give the most stable system.

We carried out calculations on: (i) a cluster of 105 atoms; (ii) a cluster of 125 atoms; and (iii) a two-cluster system of 162 atoms which partially takes into account the effects of inter-cluster interactions.

Our 105 atom cluster calculations show that the atoms in the ideal icosahedral positions give stable structure though the energy differences are only marginal (about $1.7 \mathrm{mRy} \cdot$ per atom). The calculated total energies for three different positions of atoms are given in table 1 .

In the 125 atom calculations, only the glue atoms were moved in a molecular dynamic simulations. The cage of 101 atoms was not allowed to move from the ideal icosahedral position during these simulations. We find
Table 1. Total energies of a cluster of 105-atom at different positions of atoms by density based MD simulations.

\begin{tabular}{lc}
\hline Position of atoms & Total energy \\
\hline Ideal icosahedral positions & $-230.280 \mathrm{Ry}$ \\
Crystal approximant positions & $-230 \cdot 102 \mathrm{Ry}$ \\
Penrose tiling & $-229.127 \mathrm{Ry}$ \\
\hline
\end{tabular}

that the distances of the glue atoms from the central atom have changed randomly from the initial values. We are studying in more detail, the effects of temperature on these results.

In the calculations with two clusters, only the static atom calculations were carried out for the total energies. The cages were always kept at the ideal icosahedral positions, and the glue atoms were considered at (i) ideal positions, (ii) crystal approximant positions (Guryan et al 1988; Henley and Elser 1986), (iii) a few intermediate positions, and (iv) a few extrapolated deviations from positions (i) to (ii). We found that neither the ideal nor the crystal approximant positions of glue atoms give the lowest energy though the differences are only of the order of a few mRy. These results support the recent model by Chidambaram (1999) for quasicrystals based on interacting clusters i.e. the clusters with ideal icosahedral symmetry force the 5-fold rotational symmetry on the overall structure, the glue atoms may not adhere to the long range rotational symmetry; and in the process they might as well get configurationally disordered in filling the inter-cluster regions.

\section{Acknowledgements}

The author thanks Drs R Chidambaram, S K Sikka and R S Rao for collaboration and helpful suggestions. He also acknowledges Z Hossain, R Nagarajan, L C Gupta, R Vijayaraghavan, D G Kanhere, $B$ Palanivel and $P$ Ravindran for fruitful collaboration in some of the works presented here.

\section{References}

Akahama Y, Kobayashi M and Kawamura H 1991 in Recent trends in high pressure research (ed.) A K Singh (New York: Interscience) p. 131

Alleno E, Neumeier J J, Thompson J D, Canfield P C and Cho B K 1995 Physica C229 169

Anderson J R, Papaconstantopoulos D A and Schirber J E 1981 Phys. Rev. B24 6790

Andersen O K 1975 Phys. Rev. B12 3060

Andersen O K and Jepsen O 1984 Phys. Rev. Lett. 532571

Bachelet G B, Hamann D R and Schluter M 1982 Phys. Rev. B26 4199

Car R and Parrinello M 1985 Phys. Rev. Lett. 552471

Cava R J et al 1994 Nature 367252

Chidambaram R 1996 J. Indian Inst. Sci. 76437

Chidambaram R 1999 (to be published) 
Christensen N E 1985 Phys. Rev. B32 207

Dagens L 1978 J. Phys. F Metal Phys. 82093

Descotes L and Bichara C 1995 J. Non-cryst. Solids 192-193 627

Fast L, Ahuja R, Nordstrom L, Wills J M, Johansson B and Eriksson O 1997 Phys. Rev. Lett. 792301

Focher P, Chiarotti G L, Bernasconi M, Tosatti E and Parrinello M 1994 Europhys. Lett. 36345

Godwal B K 1983 Phys. Rev. A28 1103

Godwal B K 1995a Curr. Sci. 681087

Godwal B K 1995b From astronomy to astrophysics (eds) B Sinha and $M$ Bhattacharya (Calcutta: Saha Institute of Nuclear Physics) p. 85

Godwal B K, Ng A and Dasilva L 1990 Phys. Lett. A14426

Godwal B K, Sikka S K and Chidambaram R 1979 Phys. Rev. B20 2362

Godwal B K, Sikka S K and Chidambaram R 1981 Phys. Rev. Lett. 471144

Godwal B K, Sikka S K and Chidambaram R 1983 Phys. Rep. 102121

Godwal B K, Jayaraman A, Meenakshi S, Rao R S, Sikka S K and Vijayakumar V 1997a Phys. Rev. B56 14871

Godwal B K, Rao R S, Sikka S K and Chidambaram R 1997b in Advances in high pressure research in condensed matter (eds) S K Sikka, S C Gupta and B K Godwal (New Delhi: Natl. Inst. of Sci. Commun.) p. 45

Godwal B K, Meenakshi S and Rao R S 1997c Phys. Rev. B56 14871

Guryan C A, Stephens P W, Goldman A I and Gayle F W 1988 Phys. Rev. B37 8495

Hedin L and Lundquist B I 1971 J. Phys. C4 2064

Hedin L and Lundquist B I 1972 J. Phys. C5 1629

Henley C L and Elser V 1986 Philos. Mag. B53 L59

Jagadeesh B S, Rao R S and Godwal B K 1996 in High performance computing (eds) S Sahni, V K Prasanna and V P Bhatkar (New Delhi: Tata McGraw-Hill) p. 175

Jayaraman A 1983 Rev. Mod. Phys. 5565

Jeanloz R 1987 J. Geophys. Res. 9210352

Johansson B, Ahuja R, Eriksson O and Wills J M 1995 Phys. Rev. Lett. 75280

Kagan Yu, Pushkarev V V and Holas A 1983 Zh. Eksp. Teor. Fiz. 841494 (Sov. Phys. JETP 57 870)

Kinslow R (ed.) $1970 \mathrm{High}$ velocity impact phenomena (NY: Academic Press)

Kirkpatrick S, Gelatt Jr. G D and Vecchi M P 1983 Science 220 671

Klotz S, Braden M and Besson J M 1998 Phys. Rev. Lett. 81 1239

Koehnlein D 1968 Z. Phys. 208142

Kohn W 1959 Phys. Rev. Lett. 2393

Kohn W and Sham LJ 1965 Phys. Rev. 140 A1133

Lifshitz I M 1960 Sov. Phys. JETP 111130 [J. Expt. Theor. Phys. 38 1569]

Lynch R W and Drickamer H G 1965 J. Phys. Chem. Solids 26 63

Mao H K and Hemley R 1994 Rev. Mod. Phys. 66671

Mattheiss L F 1970 Phys. Rev. B1 373

Mattheiss L F, Siegrist T and Cava R J 1994 Solid State Commun. 91587

Mazumdar C, Nagarajan R and Godart C 1993 Solid State Commun. 87413
McCarthy S L 1965 Lawrence Livermore Laboratory Report UCRL p. 4364

Meenakshi S, Vijayakumar V, Godwal B K and Sikka S K 1992 Phys. Rev. 4614359

Meenakshi S, Vijayakumar V, Godwal B K, Sikka S K, Hossain Z, Nagarajan R, Gupta L C and Vijayaraghavan R 1996 Physica B223 \& 22493

Meenakshi S et al 1998 Phys. Rev. B58 3377

Methfessel M 1988 Phys. Rev. B38 1537

Morgan J G, Von Dreele R B, Wochner P and Shapiro S M 1996 Phys. Rev. B54 812

Nagarajan R et al 1994 Phys. Rev. Lett. 72274

Novikov D L, Freeman A J, Christensen N E, Svane A and Rodriguez C O 1997 Phys. Rev. B56 7206

Palanivel B, Rao R S and Godwal B K 1999 (to be published)

Pearson M, Smargiassi E and Madden P A 1993 J. Phys. Condens. Matter 53221

Perdue J P, Burke K and Ernzerhof M 1996 Phys. Rev. Lett. 77 3865

Potzel W, Steiner M, Karzel H, Schiessl W, Kofferlein M, Kalvius G M and Blaha P 1995 Phys. Rev. Lett. 741139

Rao R S, Godwal B K and Sikka S K 1992 Phys. Rev. B46 5780

Rao R S, Godwal B K and Sikka S K 1994 Phys. Rev. B50 15632

Rose J H, Smith J R, Guinea F and Ferrante J 1984 Phys. Rev. B29 2963

Rouse C A (ed.) 1971 Prog. high temp. phys. and chem. (New York and London: Pergamon Press) Vol. 4, p. 139

Schmidt H and Braun H F 1994 Physica C229 315

Schulte O, Nikolaenko A and Holzapfel W B 1991 High Pressure Res. 6169

Shah V, Nehete D and Kanhere D G 1994 J. Phys.: Condens. Matter 610773

Sikka S K, Godwal B K and Chidambaram R 1997 in High pressure shock compression of condensed matter (eds) Davison and Sahinpoor (New York, Berlin, Heidelberg: Springer Verlag) p. 1

Sikka S K 1988 Phys. Rev. B38 8463

Skriver H L 1984 The LMTO method (Berlin: Springer)

Smargiassi E and Madden P A 1994 Phys. Rev. B49 5220

Steiner M, Potzel W, Karzel H, Schiessl W, Kofferlein M, Kalvius G M and Blaha P 1996 J. Phys.: Condens. Matter 83581

Storm A R, Wernick J H and Jayaraman A 1996 J. Phys. Chem. Solids 271227

Struzhkin V V, Timofeev Y A, Hemley R J and Mao H 1997 Phys. Rev. Lett. 214262

Takemura K 1995 Phys. Rev. Lett. 751807

Takemura K 1997 Phys. Rev. B56 5170

Vijayakumar V, Rao R S and Godwal B K 1997 in Advances in high pressure research in condensed matter (eds) S K Sikka, S C Gupta and B K Godwal (New Delhi: Natl. Inst. of Sci. Commun.) p. 318

Vohra Y K and Akella J 1991 Phys. Rev. Lett. 673563

Vohra Y K and Holzapfel W B 1993 High Pressure Res. 11223

Volkov A P, Voloshin N P, Vladimira A S, Nogin V N and Simonenko V A 1980 Pls'ma Zh. Eksp. Teor. Fiz. 31 623; JETP Lett. 31588 (1980)

Wills J M and Cooper B R 1987 Phys. Rev. B36 3809

Zel'dovich Ya B and Raizer Yu P 1967 Physics of shock waves and high temperature hydrodynamic phenomena (New York: Academic Press) Vols 1 \& 2 